\title{
Relative income change and pro-poor growth
}

\author{
Marek Kośny ${ }^{1}$ - Gastón Yalonetzky²
}

Received: 3 July 2014/Accepted: 30 July 2015/Published online: 13 August 2015

(C) The Author(s) 2015. This article is published with open access at Springerlink.com

\begin{abstract}
Most methods for the analysis of distributional change rely on the changes in the income of a particular group of people, taking either the situation of this group in the previous period, or the average change in the population, as reference point. By contrast, we propose a measure of distributional change based on the comparison of a group's wellbeing standard against a richer group, thereby capturing a notion of change in relative income, which embodies the influence of others' wellbeing on the judgment of the group's own situation. The indices, and related relative income change (RIC) curves, are based on ratios of generalized mean incomes between two groups, which render them closely related to Zenga's inequality measures and Lorenz curve comparisons, when arithmetic means are used. We consider both relative and absolute income cut-offs for the group partitions. Differences and similarities between our measures of RIC and the Growth Incidence Curve are also discussed. These are highlighted in an empirical illustration on European countries.
\end{abstract}

Keywords Relative income - Income distribution · Pro-poor growth

JEL Classification $\mathrm{I} 31 \cdot \mathrm{J} 30$

Marek Kośny

marek.kosny@ue.wroc.pl

Gastón Yalonetzky

g.yalonetzky@leeds.ac.uk

1 Institute of Applied Mathematics, Wroclaw University of Economics, ul. Komandorska 118/120, 53-345 Wrocław, Poland

2 Leeds University Business School, The University of Leeds, Leeds LS2 9JT, UK 


\section{Introduction}

Analyzing the pattern of changes in income distributions provide further knowledge as to the effects of economic processes. Above and beyond stating whether the level of income inequality has increased or decreased, the main purpose of these analyses is to identify areas in the distribution of income in which changes of a given type have occurred. Of particular interest in these studies is the situation of the poorwhether (and how) their incomes grow, and whether economic growth is "propoor" or not (see for instance Kakwani and Pernia 2000; Dollar and Kraay 2002; Kraay 2006; Son and Kakwani 2008; Foster and Szekely, 2008; Deutsch and Silber 2011; Ferreira, 2012). Yet an identification of the pattern of changes in the distribution of income is not limited to the area of low incomes. No less important are other areas, including the richest and the middle class. Arguably, their mutual relations can affect the stability of society, and the prospects of social tension (e.g. see Acemoglu and Robinson 2009; Esteban and Ray 2011).

Assessing a pattern of changes in the distribution of income depends on the perception of changes in the level of income. Among the proposed methodologies, two main streams can be identified: the relative and the absolute approach (see, respectively, Ravallion 2004; Duclos 2009). Broadly speaking, they are distinguished with respect to the point of reference used for the assessment of change in income: whether absolute or relative growth is required for assessing the change as beneficial for a given group. This distinction is closely related to the way poverty and inequality are measured. When an analysis is concentrated on satisfying basic needs, absolute growth of income is worth considering. This is of primary concern for the poorest countries, where a large proportion of the population receives an income below the subsistence minimum (see Duclos 2009, p. 38). Relative dependencies gain importance in the case of developed countries (Layard et al. 2010). Ensuring their own physical existence still remains the most important issue for a certain group of the poorest. But the assessment of an individual's (or household's) own relative situation, in reference to the situation of others, becomes an important factor.

This direct assessment of the relative situation of different income groups is the basis for the measures proposed in this paper; by contrast to other measures commonly used in empirical analyses. Our measures do not describe an income change at a particular position of the income distribution (as, for example, in the case of the Growth Incidence Curve (see Ravallion and Chen 2003), which assesses the relative change in income for a given quantile) or a change in income of a group of people in comparison to the average change in the overall population (see, for example, second-order pro-poorness; Duclos 2009, p. 50). Our proposed measures reflect the change in the relative situation of two groups, telling us whether the situation of the first of them has improved relative to the other. We argue that this approach helps to incorporate interactions between these groups. The increase in income of any person has an impact on the others, especially groups with similar incomes. For example, if people's own welfare assessments take into account the situation of others, then, arguably, the relative situation of the poorer group 
(especially slightly poorer) deteriorates in the case of an income increase in the richer group. In this framework, an increase in the income of the poorer is, in fact, of relatively lesser worth, if the income of someone wealthier increased much more. Such a relative assessment of groups' situation is of particular importance in the case of countries with a high level of income inequality, but has also been emphasized as a potential problem in the form of competitive consumerism and its effect on subjective wellbeing (e.g. see Layard 2011). Additionally, an improvement in the situation of a group of the richest people, in comparison with the poorest, but also those with an average income, could mean the risk of rising social tensions and a decrease in the stability of the existing system (e.g. see Acemoglu and Robinson 2009).

Focusing on groups of people is a key feature of our proposed measures. In contrast to analyses based on the Growth Incidence Curve, where only the situation of individuals corresponding to quantiles of a given order is actually considered, the proposed approach is based on a direct comparison of the situation of groups, which can be explicitly defined. In the case of the analysis of the relative situation of the poor, the compared groups are the poor and the non-poor. However, these groups can be distinguished within the population from the point of view of any criteria: if adequately defined, they may, in particular, reflect the sociological concept of reference groups. This group-oriented approach does not necessarily mean a lack of interest in the income distribution inside these groups. Admittedly, we may want to account for the problem of "distribution of poverty" in our assessment of a growth pattern. An equal treatment of poor people in every position in the income distribution would implicitly assume a "linear" and continuous nature of poverty, but the intensity of the poverty experience does not have to be a linear function of a poverty gap. Despite an arbitrary (contractual) character of poverty lines, ${ }^{1}$ one could posit the existence of some thresholds below which living standards decrease substantially. This actual nature of poverty is recognized in an extension to our proposed measure of a relative income change (RIC), in which we weigh poor incomes in a manner akin to Atkinson's inequality measures based on generalized means (Atkinson 1970). As this procedure prioritizes the wellbeing of the poorest, the proposed measures relate to the concept of inclusive growth (see Ali and Son 2007 , p. 12), the inability to participate in subsequent aspects of social life constitutes the thresholds discussed above. ${ }^{2}$

Our measure of RIC computes the difference in relative income between two periods, where the relative income is defined by the income of the poor divided by the income of the non-poor, and the two groups are defined by a percentile threshold. Additionally, we propose a RIC curve which plots the RIC index against each of the possible group-splitting percentiles. We study the properties of both the

\footnotetext{
1 Poverty lines are defined arbitrarily - both if they are related to some summary statistics of income in the population (e.g. mean or median) and when they are defined as absolute values. A subsistence minimum can be given as an example of the latter. According to the definition of this scale, nobody with a lower income should survive. Therefore, accounting for the sense of poverty is given in the form of quasicontinuous weights, not defined for thresholds.

${ }^{2}$ Kakwani and Son (2008) also weigh income growth rates by measures of poverty intensity to construct their poverty-equivalent growth rates.
} 
RIC measure and the RIC curve, and find that the latter's behaviour is closely related to the Lorenz inequality ordering. Likewise, our RIC curve is closely related to Zenga's family of inequality indices (Zenga 2007).

The paper is organized as follows. Section 2 introduces our measures of RIC, followed by the RIC curves, an extension emphasizing the experience of the poorest among the poor, and an extension based on alternative definitions of the groups. We include a thorough discussion around the measures' and curve's fulfilment of desirable properties. Section 3 outlines the similarities and differences between the RIC framework and related methods. Section 4 develops the empirical illustration carried out for three groups of European countries. Section 5 concludes.

\section{Measure of relative income change}

Let $x(p, t)$ be the income quantile for percentile $0 \leq p \leq 1$ in period $t^{3}$ With $p$ we can split the population into two contiguous, non-overlapping groups: a bottom group (with incomes $x(q, t)$ such that $0 \leq q \leq p$ and a top group (with with incomes $x(q, t)$ such that $p<q \leq 1$. Then we can define, respectively, the bottom and the top means:

$$
\begin{gathered}
\underline{\mu}(p, t)=\frac{1}{p} \int_{0}^{p} x(q, t) d q \\
\bar{\mu}(p, t)=\frac{1}{1-p} \int_{p}^{1} x(q, t) d q .
\end{gathered}
$$

Then our measure of RIC between periods $t$ and $\tau$ as defined as follows:

$$
R(p ; t, \tau)=\frac{\underline{\mu}(p, t)}{\bar{\mu}(p, t)}-\frac{\mu}{\bar{\mu}(p, \tau)}
$$

Note that $p$ the relative size of the poorer group, remains the same across periods. Hence we are not using, for instance, an absolute poverty line to divide the two groups in either period. We will consider this alternative as an extension in a section below.

$R(p ; t, \tau)$ quantifies the absolute change in the average income of the poorer group measured in relative terms as a ratio of the richer group's average income. Besides, as long as $\exists p \mid x(p, t)>0 \forall t, \tau$ then clearly $-1 \leq R(p ; t, \tau) \leq 1$. Positive values of $R$ indicate an improvement in the relative situation of the poorer group. By contrast, being a relative measure, $R$ holds no information regarding the absolute situation of both groups. This means that even in the case of a decrease in overall

\footnotetext{
${ }^{3}$ In the next section of the paper, income will be used as a proxy for welfare. But it could be replaced with expenditure or any other measure of welfare (see Slesnick 1998). On a broader discussion on the application of non-income variables see Grosse et al. (2008).
} 
income, the change can be considered favourable to the poor, e.g. if the decline among the richer is starker.

The behaviour of $R(p ; t, \tau)$ can be further described by listing a set of properties fulfilled by the index:

Axiom 1 Scale invariance: if every value of $x$ s multiplied by the same scalar $\lambda>0$ in both periods $t$ and $\tau$ then the value of $R(p ; t, \tau)$ remains unaffected.

Axiom 2 Population principle: Replicating every individual by the same number in both periods does not change the value of $R(p ; t, \tau)$.

Axiom 3 Anonymity: No information except for the income is taken into account when assessing the distributional change.

Axiom 4 Focused monotonicity: $R(p ; t, \tau)>0$ if a person who remains in the poorer group in both periods experiences an increase in income between $t$ and $\tau$.

Axiom 5 Sensitivity to Pigou-Dalton transfers between individuals from different groups: $R(p ; t, \tau)>0$ if distribution $t$ is obtained from distribution $\tau$ by a PigouDalton transfer involving a person in percentile $q>p$ in period $\tau$ together with a person in percentile $r<p$ in the same period. ${ }^{4}$

Axiom 6 Insensitivity to Pigou-Dalton transfers within individuals belonging in the same group: $R(p ; t, \tau)=0$ if distribution $t$ is obtained from distribution $\tau$ by a Pigou-Dalton transfer involving a person in percentile $q$ in period $\tau$ together with a person in percentile $r$ in the same period, such that either $q \leq p \wedge r \leq p$ or $q>p \wedge r>p$.

Axiom 4 through 6 summarize the main traits of sensitivity to distributional change exhibited by $R . \quad R>0$ whenever people from the richer group transfer income to people in the poorer group between the two period; whereas $R$ is insensitive to similar transfers when the two people involved belong to the same group. In the extensions further below we propose a modified version of $R$ which is also sensitive to intra-group transfers in order to: (1) prioritize the wellbeing of the poorest among the poor; and (2) deprioritize the wellbeing of the richest among the rich.

\subsection{The RIC curve}

If we plot $R(p ; t, \tau)$ against $p$ across its whole domain we derive the RIC curve. Like the Growth Incidence Curve the RIC curve is helpful in the analysis of pro-poor growth. In particular, the RIC curve enables detection of the size of the bottom segments of the population that benefit the most and least vis-a-vis their richer complementary counterparts. The differences between the Growth Incidence Curve and the RIC curve will be explored further in Sect. 3.

\footnotetext{
${ }^{4}$ Note that $R$ fulfills this property even when one of the people involved changes group status in period $t$ as a consequence of the income transfer.
} 
The RIC curve satisfies axioms of scale invariance, anonymity and population principle. In relation to the above axioms 5-6, the RIC curve behaves the following way:

Axiom 7 Sensitivity to Pigou-Dalton transfers (between individuals from either different groups or within the same group): $R(p ; t, \tau) \geq 0 \forall p \wedge \exists p \mid R(p ; t, \tau)>0$ if distribution $t$ is obtained from distribution $\tau$ by a Pigou-Dalton transfer.

Note that the RIC curve does not satisfy the axiom of focused monotonicity since, depending on the choice of $p$, the same individual experiencing and increase in income may find himself either in the bottom group or in the top one. Note also that axiom 7 is coherent with axioms 5 and 6. That is, when the Pigou-Dalton transfer involves percentiles $q<r$ then, $R(p ; t, \tau)=0 \forall p<q \vee p>r \quad$ and $R(p ; t, \tau)>0 \forall q<p<r$. In fact, whenever $R(p ; t, \tau) \geq 0 \forall p \wedge \exists p \mid R(p ; t, \tau)>0$, i.e. when the RIC curve is never negative and at least positive for one value of $p$, then distribution $t$ is less unequal than distribution $\tau$ according to any Lorenz-consistent inequality index $I$. As the following proposition states:

Proposition $1 \quad R(p ; t, \tau) \geq 0 \forall p \wedge \exists p \mid R(p ; t, \tau)>0$ if and only if $I(t)<I(\tau)$ or any Lorenz-consistent inequality index $I$.

Proof Let $L(p, t) \equiv \frac{1}{\mu(t)} \int_{0}^{p} x(q, t) d q$ be the Lorenz curve of distribution $t$ evaluated at percentile $p$, with $\mu(t)$ being the mean of distribution $t$. Then it is straightforward to show that: $R(p ; t, \tau)=\left[\frac{1-p}{p}\right]\left[\frac{L(p, t)}{1-L(p, t)}-\frac{L(p, \tau)}{1-L(p, \tau)}\right]$. Hence $R(p ; t, \tau) \geq 0 \forall p \wedge$ $\exists p \mid R(p ; t, \tau)>0$ if and only if $L(p, t)-L(p, \tau) \geq 0 \forall p \wedge \exists p \mid L(p, t)-L(p, \tau)>0$ i.e. if there is Lorenz dominance of $t$ over $\tau$ which implies, and is implied by, $I(t)<I(\tau)$ or any Lorenz-consistent inequality index $I$.

\subsection{Extensions: prioritization of the poorest}

Placing a higher weight on the incomes of the poorest among the poor is a common trait of many poverty measures, including the Watts index and any member of the Foster-Greer-Thorbecke family (Foster et al. 1984) in which the normalized poverty gaps are raised to a power higher than one. This way of proceeding is meant to capture several related aspects of the poverty experience, including low incomes limiting or even precluding participation in a social life, negative impacts on health status due to lack of healthcare and proper food, etc.

While an increase in the income of the poorest is unconditionally considered propoor, in the case of other distributional changes the answer does not need to be so obvious. For example, the poorest can feel more deprived as a result of the increase in the income of "the richer poor" (who are still poor after the change). Moreover, such a change in the distribution of income in some situations may cause an absolute worsening of the situation of the poorest, the growth of the purchasing power of the poor as a whole can result in an increase in prices of some basic goods. Such mechanisms, however, are not necessarily reflected in the usual measures. 
In order to prioritise the situation of the poorest members of the population, we propose a weighted analogue of $R$ relying on generalized means. Hence we follow an approach akin to Foster and Szekely (2008), which in turn draws inspiration from Atkinson's inequality indices (Atkinson 1970). For this purpose we start by defining the bottom and top generalized means:

$$
\begin{gathered}
\underline{\mu}(p, t ; \alpha)=\left[\frac{1}{p} \int_{0}^{p} x(q, t)^{\alpha} d q\right]^{\frac{1}{\alpha}}, \quad \forall \alpha \neq 0 \\
\underline{\mu}(p, t ; 0)=\frac{1}{p} \int_{0}^{p} \log x(q, t) d q \\
\bar{\mu}(p, t ; \beta)=\left[\frac{1}{1-p} \int_{p}^{1} x(q, t)^{\beta} d q\right]_{p}^{\frac{1}{\beta}} \forall \beta \neq 0 \\
\bar{\mu}(p, t ; 0)=\frac{1}{1-p} \int_{p}^{1} \log x(q, t) d q
\end{gathered}
$$

Then we redefine the measure of RIC as a function of the two parameters $\alpha$ and $\beta$ :

$$
R(p ; t, \tau ; \alpha, \beta)=\frac{\underline{\mu}(p, t ; \alpha)}{\bar{\mu}(p, t ; \beta)}-\frac{\mu}{\bar{\mu}(p, \tau ; \tau ; \beta)}
$$

Now we consider the following axiom of prioritization of the poorest among the poor:

Axiom 8 Prioritization of the poorest among the poor: $R(p ; t, \tau ; \alpha, \beta)>0$ if distribution $t$ is obtained from distribution $\tau$ by a Pigou-Dalton transfer involving a person in percentile $q<p$ in period $\tau$ together with a person in percentile $r<p$ in the same period.

It turns out that we can derive a whole family of RIC measures which place higher weight on the poorest incomes by focusing on the indices $R(p ; t, \tau ; \alpha, 1)$, i.e. with $\beta=1$. Proposition 2 identifies the sub-domain of $\alpha$ or which $R(p ; t, \tau ; \alpha, 1)$ satisfies axiom 8 , in addition to axioms 1 through 5 , plus an amended version of axiom 6 (since now $R(p ; t, \tau ; \alpha, 1)$ is only insensitive to within-group Pigou-Dalton transfers among the richer group):

Proposition $2 R(p ; t, \tau ; \alpha, 1)$ satisfies axiom 8 if and only if $\alpha<1$. The proof is straightforward. 


\subsection{Extensions: prioritization of the poorest and de-prioritization of the richest}

We can also combine a prioritization of the poorest among the relatively poor group with a de-prioritization of the wealthiest among the relatively rich group. With this purpose we complement axiom 8 with axiom 9:

Axiom 9 De-prioritization of the wealthiest among the rich: $R(p ; t, \tau ; \alpha, \beta)>0$ if distribution $t$ is obtained from distribution $\tau$ by a Pigou-Dalton transfer involving a person in percentile $q>p$ in period $\tau$ together with a person in percentile $r>p$ in the same period.

Finally, Proposition 3 identifies the sub-domains of $\alpha$ and $\beta$ for which $R(p ; t, \tau ; \alpha, \beta)$ satisfies axioms 8 and 9 , in addition to axioms 1 through 5. Now the combined fulfilment of axioms 8 and 9 replaces the satisfaction of axiom 6 . In other words, axiom 6 is incompatible with the joint axioms 8 and 9:

Proposition $3 R(p ; t, \tau ; \alpha, \beta)$ satisfies axioms 8 and 9 if and only if $\alpha<1<\beta$. The proof is straightforward.

\subsection{Extensions: alternative divisions of the groups}

In the previous sections our RIC measures define the poor and the non-poor in relative terms, by setting a percentile cut-off. Alternatively we can also identify the poor and the non-poor in absolute terms, using a predetermined poverty line, $z$, from the admissible domain of income $\mathfrak{D}$. Then we can construct measures akin to the aforementioned. However, generally, the measures based on absolute poverty lines will differ, from those based on percentile cut-offs, in that their value does not only depend on the changes in the income shares of the two groups, as is the case of $R(p ; t, \tau)$ in (1) for instance, but also on the changes in the poverty and non-poverty headcounts.

For example, we can propose an index $R(z ; t, \tau)$ is an alternative to $R(p ; t, \tau)$. In order to do so, firstly, we define the poverty headcount in period $t$ : $h(z, t) \equiv \operatorname{Pr}[x(t) \leq z]$. We also need to redefine the bottom and top means in terms of the headcounts:

$$
\begin{gathered}
\underline{\mu}(z, t)=\frac{1}{h(z, t)} \int_{0}^{h(z, t)} x(q, t) d q \\
\bar{\mu}(z, t)=\frac{1}{1-h(z, t)} \int_{h(z, t)}^{1} x(q, t) d q
\end{gathered}
$$

Finally, we define the index:

$$
R(z ; t, \tau)=\frac{\mu}{\bar{\mu}(z, t)}-\frac{\mu}{\bar{\mu}(z, \tau)}
$$


It is easy to show that $R(z ; t, \tau)$ satisfies the same axioms as $R(p ; t, \tau)$. Moreover we can define a similar RIC curve by plotting $R(z ; t, \tau)$ gainst the domain of $z$ and derive results similar to Proposition 1. Perhaps more interesting in $R(z ; t, \tau)$ is its straightforward decomposition into changes in the share of income accruing to the poor and changes in the poverty headcount:

$$
R(z ; t, \tau)=\bar{H} \Delta S+\bar{S} \Delta H,
$$

where:

(a) $\Delta S \equiv\left[\frac{s(z, t)}{1-s(z, t)}-\frac{s(z, \tau)}{1-s(z, \tau)}\right]$ and $s(z, t)$ is the share of total income held by the poor in period $t$.

(b)

$$
\Delta H \equiv\left[\frac{1-h(z, t)}{h(z, t)}-\frac{1-h(z, \tau)}{h(z, \tau)}\right]
$$

(c)

$$
\bar{H} \equiv \frac{1}{2}\left[\frac{1-h(z, t)}{h(z, t)}+\frac{1-h(z, \tau)}{h(z, \tau)}\right]
$$

(d)

$$
\bar{S} \equiv \frac{1}{2}\left[\frac{s(z, t)}{1-s(z, t)}+\frac{s(z, \tau)}{1-s(z, \tau)}\right] .
$$

Since $R(z ; t, \tau)$ satisfies the same axioms as $R(p ; t, \tau)$, when we use absolute poverty lines then we can relate improvements in our measure of pro-poor growth based on changes in relative income share to two key components: changes in the poverty headcount and changes in the share of income held by the poor. Ceteris paribus, any reduction in the proportion of poor people increases the value of $R(z ; t, \tau)$, thereby reflecting a pro-poor growth experience. Likewise, any increase in the share of income held by the poor, ceteris paribus, produces the same effect in $R(z ; t, \tau)$. Moreover, a reduction in the proportion of poor people accompanied by an increase in the share of income held by the poor, for every choice of $z$ renders $R(z ; t, \tau)>0 \forall z$. That is, if distribution $t$ both first-order and Lorenz dominates distribution $\tau$ then the mean of the poor relative to the non-poor increases for every choice of poverty line. However the reverse is not true, i.e. $R(z ; t, \tau)$ could still be positive if either $\Delta S$ or $\Delta H$ (but not both simultaneously) is negative, as long as the other component is positive and more than offsets the effect of the negative component on $R(z ; t, \tau)$.

\section{A numerical illustration}

In order to highlight some properties and features of the proposed measures, we present a numerical hypothetical illustration in Table 1. $x_{i}^{0}$ and $x_{i}^{1}$ denote the incomes 


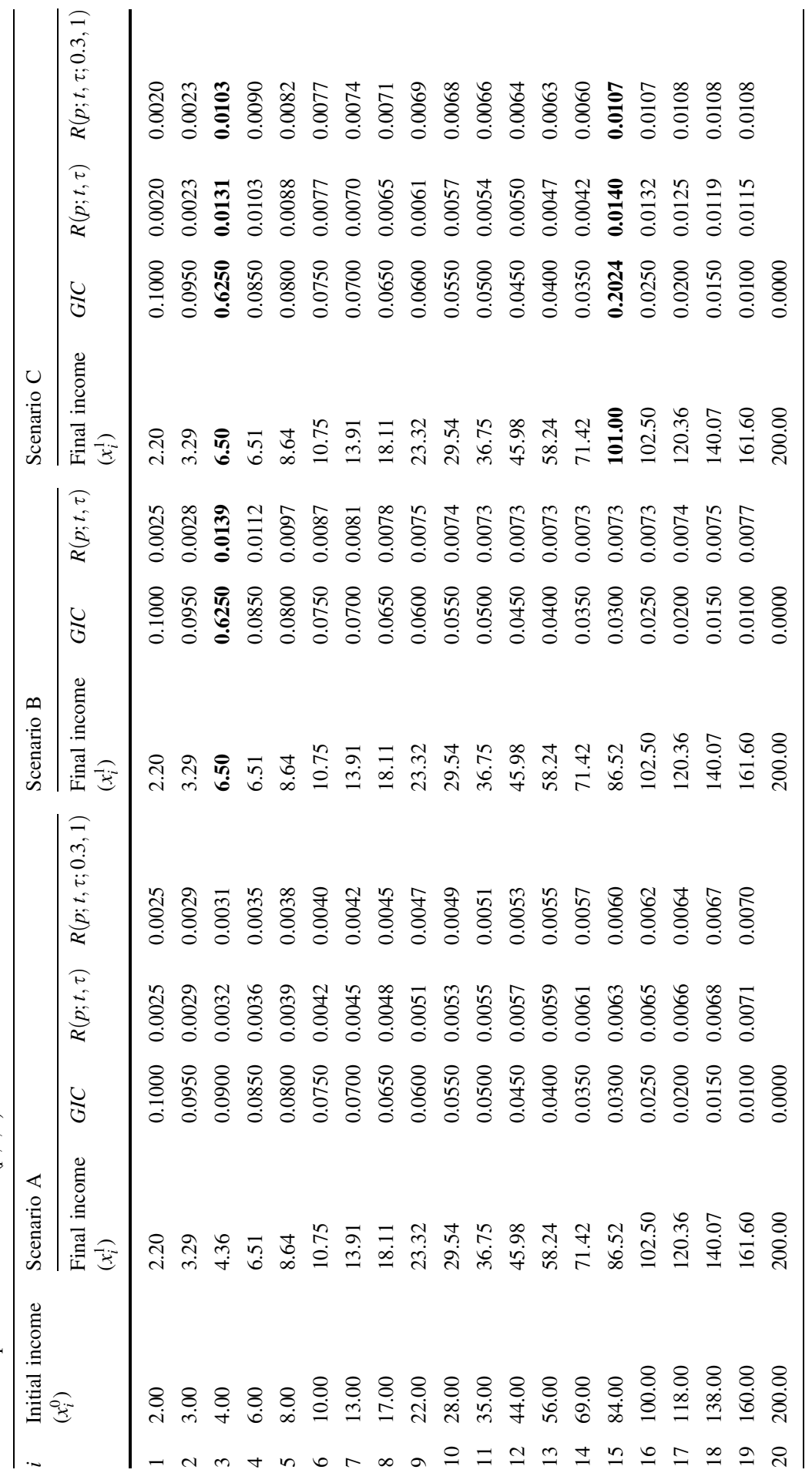



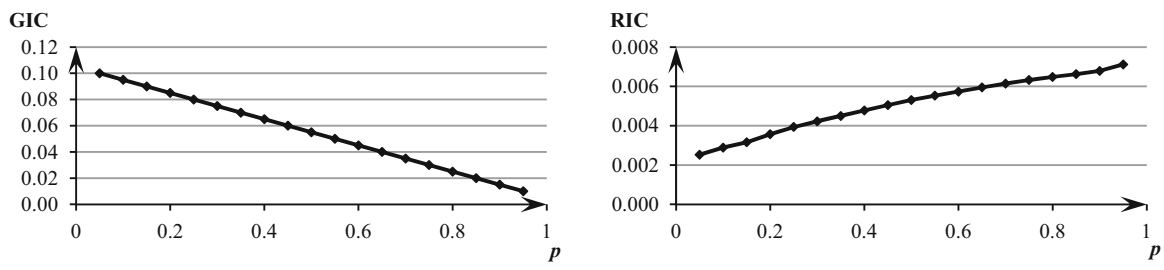

Fig. 1 GIC and RIC curves for scenario A

of person $i$ in the initial and final period, respectively. One of three scenarios of change: We consider three alternative scenarios for the final income distribution: A, B or C. Final incomes in scenarios $\mathrm{B}$ and $\mathrm{C}$ are the same as in scenario $\mathrm{A}$, with the exceptions of $i=3$ (scenarios $\mathrm{B}$ and $\mathrm{C}$ ) and $i=15$ (scenario $\mathrm{C}$ ). The differences are highlighted in bold font.

For each scenario we compute $R(p ; t, \tau)$, together with the popular Growth Income Curve (GIC; Ravallion and Chen, 2003) for comparison. For scenarios A and $\mathrm{C}$ we also report $R(p ; t, \tau ; 0.3,1)$. Their respective values are in Table 1.

Figure 1 presents the GIC and RIC curves for scenario A. The GIC shows all positive growth rates that are smaller for higher initial incomes. Naturally, the RIC describes this situation with positive and increasing values: the situation of the lower group has improved with respect to the upper group, irrespective of the percentile choice for group partition. Yet the RIC curve more directly shows that the relative benefits from income growth rise with $p$.

Figure 2 shows the RIC curves for the three scenarios:

Scenario B differs from A only in the income of $i=3$, which rises from 4 to 6.50; whereas in A income growth for $i=3$ is smaller, from 4 to 4.36. Until $i=2$ the RIC curve of $\mathrm{A}$ is slightly above $\mathrm{B}$, but then from $i=3$ onward a spike favouring $\mathrm{B}$ is prominent. This advantage over scenario A, due to the improvement in the situation of the third person, remains throughout until $p=1$, but decreases as the size of the bottom group increases (thereby rendering the income of the third person relatively less important in the computation of the overall mean).

Scenario C differs from B only in the income of $i=15$ (101 in C against 86.52 in scenarios $\mathrm{A}$ and $\mathrm{B}$, in the final period). Comparing $\mathrm{C}$ against $\mathrm{A}$, only the income growth situation of two individuals has changed, but in fact, such changes affect the situation of the others, by modifying the relative means of the two groups.

The RIC curve of $\mathrm{C}$ is above A's between roughly $p=0.2$ and $p=0.5$. During this interval, the gain in the third income contributes more to the bottom group's mean than the gain in the 15 th income does to the top group's mean. However past $p=0.5$, the relative contributions are reversed and the RIC of C falls below A's. Finally, once both the third and the 15th person belong to the poorer group (i.e. for $p>0.7$ ) the RIC curve of C remains permanently above A's. These dynamics cannot be easily observed in the individual approach implicit in the GIC curves, as it rules out any judgments of relative income or welfare measurement interactions across individuals/households. 


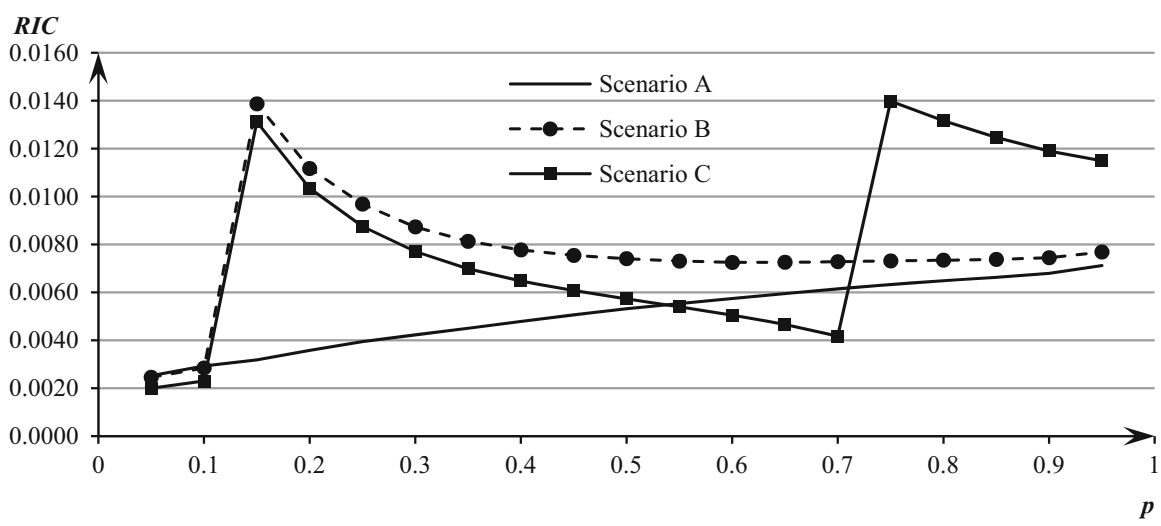

Fig. 2 RIC curves for scenarios $\mathrm{A}, \mathrm{B}$ and $\mathrm{C}$

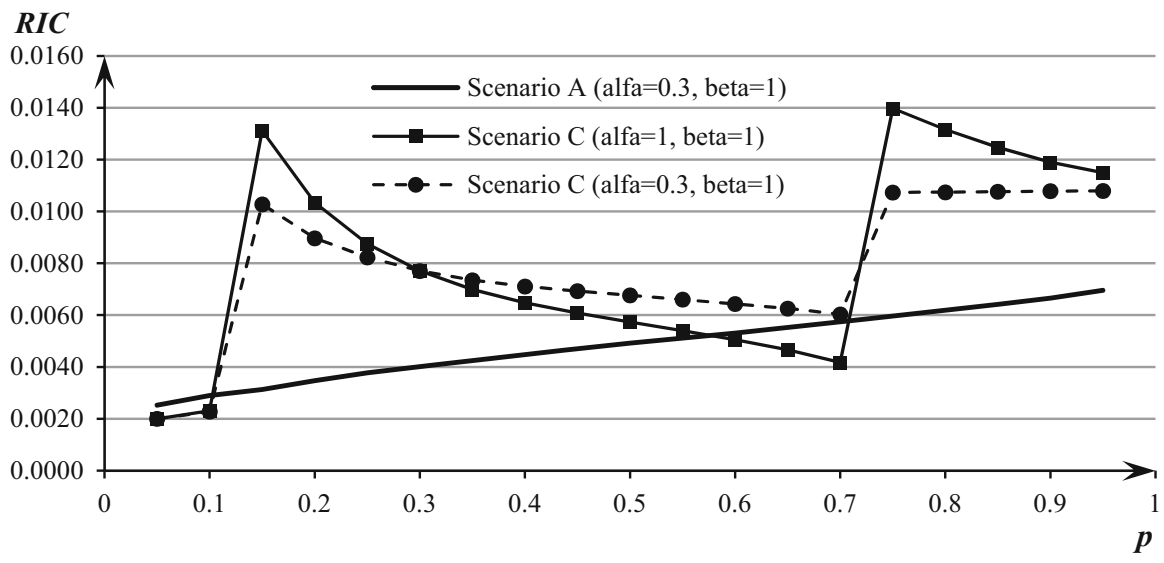

Fig. 3 RIC curves with different values of $\alpha$ for scenarios A and C

The precise scope of the changes which result from an increase in the income level of given individuals depends, naturally, on the prioritization of people within the groups. For example, Fig. 3 shows RIC curves for scenarios A and B with two choices of $\alpha$

Note how a lower choice of $\alpha$ " fflattens" the RIC curve of C. This means that the growth in the income of the third person is less important with a lower $\alpha$ when the third person is among the least poor of the poor (e.g. when $p=0.2$ ), however as more people join the ranks of the bottom group and the third person is somehow "sent to the rear", i.e. richer people join in, then the contribution of the income change of the third person increases. This latter increase is significant enough to completely offset the effect of the growth in the income of the 15th person, to the point that the RIC curve of scenario $\mathrm{C}$ with $\alpha=0.3$ is never below the RIC curve of scenario A with the same $\alpha$ (which features lower growth in the income of the 15 th person). 


\section{Empirical results for some European countries}

We now use $R(p ; t, \tau)$, along with the GIC, in order to assess the pattern of growth for some of the countries covered by the Luxembourg Income Study Database (2012). The available data generally come from national household budget surveys (except for Denmark, where the data are taken from the tax register). The harmonization of national datasets is completed before making the data available. However, full harmonization is not possible due to the differences between the analyzed countries (fiscal systems, education etc.). Such discrepancies should not, however, significantly influence the presented results because the basic comparison between the poor and the non-poor is made within the same dataset (for a given country and year).

The largest set of countries in this database is available for the so-called Wave 6 (around 2004). Wave 5 (around 2000) provides the "initial period" reference point. ${ }^{5}$ The analysis is based on disposable income. Variable dpi denotes net income (gross income minus income taxes and mandatory payroll taxes GI-(PAYROLL + V11) for gross datasets and net income GINET for net datasets) per household. Each household is assigned the person weight in order to adjust for the structure of the population and the number of individuals in the household. A detailed description of the data and the precise definitions of variables (components and structure) are provided by the LIS Data Centre on the project website: http://www.lisdatacenter. org.

GDP growth in the period under consideration was mediocre, both in advanced economies and in the whole world (see World Economic Outlook Database 2013). The burst of the "dot-com bubble" resulted in an economic slowdown after the rapid growth of the late 1990s.

The analysis was carried out taking into account the geographical location of countries (this factor was widely considered, among others, during the next crisis which began in 2008). Countries from southern and northern Europe, available in the LIS Database for selected years, were considered. Two countries from central Europe (Luxembourg and Switzerland), whose economies largely depend on the financial sector, were also included.

In the southern European countries analyzed, differences in income between the poorest (first quintile of the income distribution) and the rest of the population decreased slightly, indicating a pro-poor change. In Italy and Greece, the RIC values (for the period 2000-2004) decreased with income. This denotes the kind of change which is favourable not only for the poorest, but also for the richest in the population, as the distance between the richest and the middle-income group has increased in the period analyzed. A different pattern of changes was observed in Spain. Changes which are relatively favourable to the poorest (in relation to the rest of the population) were accompanied by a significant improvement in the relative situation of the upper-middle-income group (see the rising RIC curve for Spain in

\footnotetext{
5 Minimum 4-year distance between the beginning and the end of the analyzed period is required. Only datasets available in the LIS Web Tabulator were taken into account. We used average incomes corresponding to consecutive deciles groups.
} 

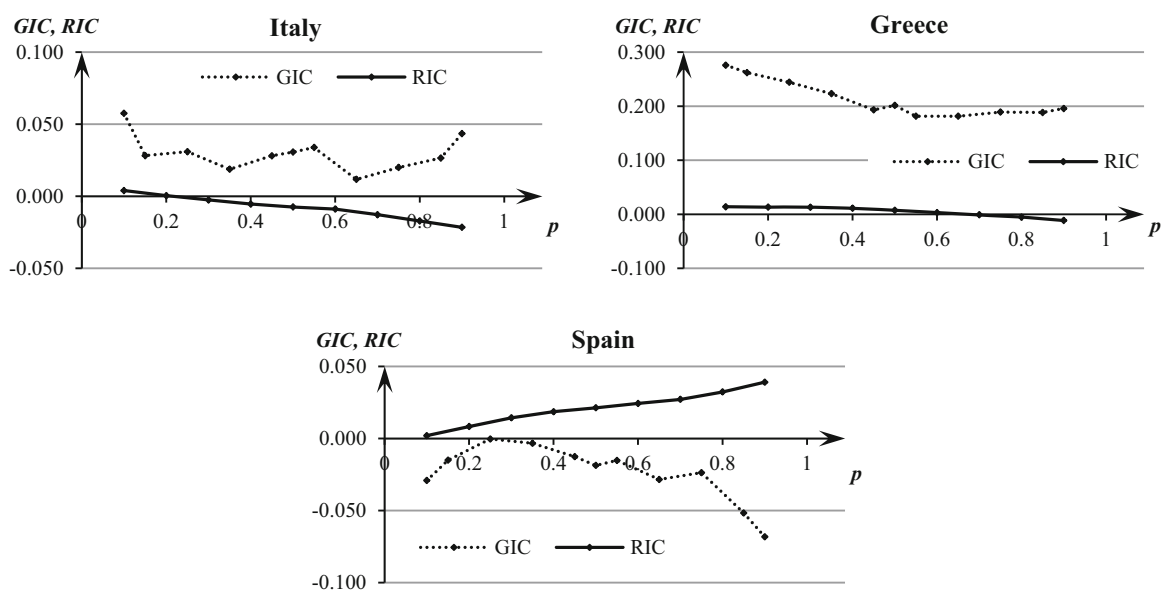

Fig. 4 GIC and RIC curves between Wave 6 (around 2004) and Wave 5 (around 2000) for three southern European countries

Fig. 4). The strictly relative nature of this improvement is emphasized by the negative values of the GIC, which means a decrease in nominal income.

A different direction of change in the situation of the poorest was observed in several northern European countries. Figure 5 shows negative values for the RIC curve in the cases of Finland, Denmark and Norway. These negative values were observed also for higher values of $p$, which indicates an increase in income inequality and the relative improvement only in the situation of the wealthiest.

The exception among the Scandinavian countries was Sweden, whose RIC curve was consistently positive. The highest values in its lower part suggest that the improvement in the relative situation was the highest for the least affluent group. In contrast to the other three country-cases, the robustly positive values of the RIC curve in Sweden denote a reduction in Lorenz-consistent inequality.

A different pattern of change was observed in the case ofLuxembourg and Switzerland, which is why they were analyzed as a separate, central European group. Their common feature is the large dependence of their economies on the financial sector. The distinctive feature of these countries (against those previously analyzed, as well as others which are available in the LIS Web Tabulator) is a local decrease in income for the group below the median (see GIC in Fig. 6). In this situation, inference about pattern of income growth on the basis of GIC can be confusing. Despite the increase in the income of the poorest groups (positive values of $G I C$ ), the overall change should be considered anti-poor (negative $R I C$ values) in relative terms. All RIC values for Luxembourg are negative and this indicates a relative improvement only in the situation of the richest, whereas $R I C$ values for Switzerland grew with the increase in income which denotes that the upper-middleincome group is the most favoured.

The results show that the use of $R I C$ curves allows a relatively unambiguous assessment of the nature of changes in the distribution of income (such an 

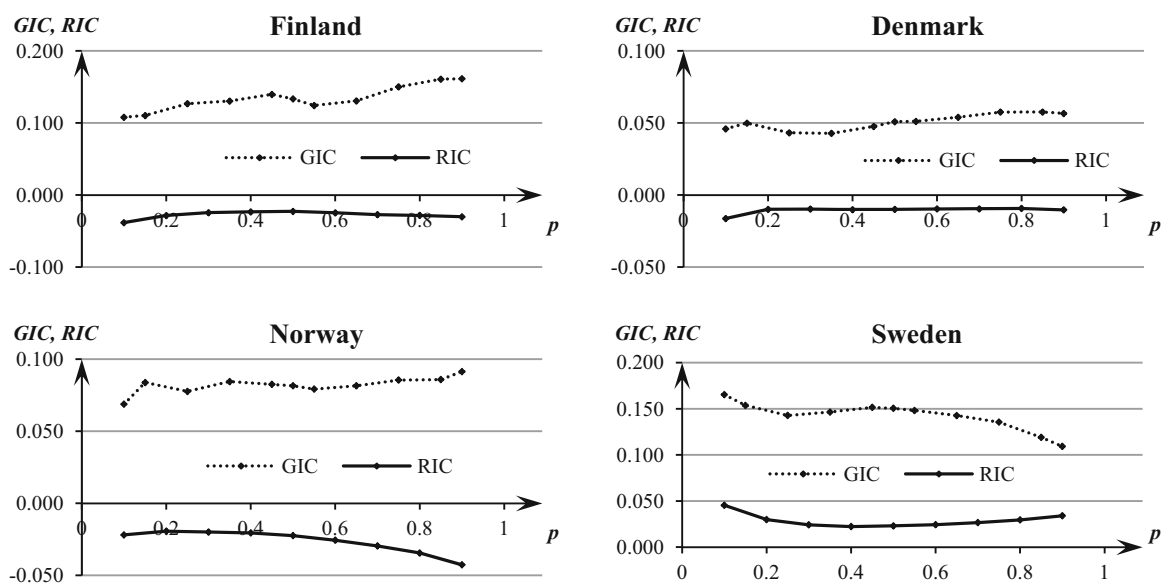

Fig. 5 GIC and RIC curves between Wave 6 (around 2004) and Wave 5 (around 2000) for northern European countries
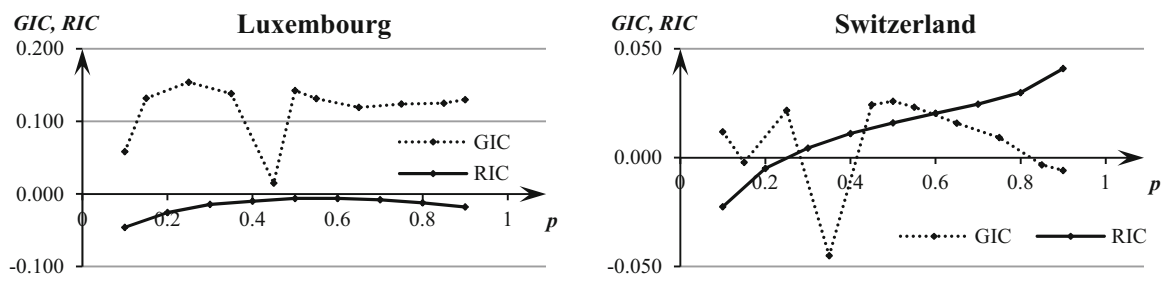

Fig. 6 GIC and RIC curves between Wave 6 (around 2004) and Wave 5 (around 2000) for Switzerland and Luxembourg

identification can be much more difficult using GIC). It also allows for the assessment of changes in the relative situation of the richer and poorer groups. We observed, for three Scandinavian countries and Luxembourg, a deterioration in the situation of the poorer compared to the wealthier individuals ( $R I C$ takes only negative values) which implies an increase in income inequality. Considering the potentially negative consequences of such changes (e.g. increase in social tensions), it is worth noting that these countries are characterized by very low levels of income inequality (according to LIS data, the value of the Gini coefficient for these countries is between 0.23 and 0.28 ), significantly lower than for the analyzed countries from the south of Europe (0.32-0.35). Verification of the hypothesis that these factors are interrelated, and periods of economic downturn are characterized by pro-poor changes in countries with a relatively higher income inequality and anti-poor changes in countries with lower income inequality, would require a much broader analysis. 


\section{Conclusion}

Identifying a pattern of changes in the income distribution can be a crucial element in the process of designing a social policy. Ideally a distributional assessment should not just comply with some formal statistical requirements, but should also reflect individuals' perceptions of distributional change including those pertaining to relative income.

The proposed relative income measures are inspired by Zenga's inequality index. The essential element of this approach is the direct comparison of income across subgroups in the population. For a pro-poor assessment, the groups are made up of the poor and the non-poor. Such a direct comparison reflects the general idea of a reference group; albeit, admittedly, in a restricted way as the basis of differentiation between groups is mainly uni-dimensional (e.g. income or another single measure of welfare). In addition to the RIC index, we introduce a RIC curve, while showing both how it relates to Lorenz inequality comparisons, and how it complements and differs from the popular Growth Incidence curve.

We also provide several interesting extensions of the relative-income-change measurement framework. Firstly, we show how the RIC indices can be amended to prioritize the poorest among the poor, thereby capturing the non-linear relationship between the poverty gap and the actual situation of the poor. Likewise we show how the RIC indices can further be amended to de-prioritize, or penalize, income gains accrued by the richest among the rich. Finally, we propose a RIC index, and curve, based on group partitions driven by absolute income cut-offs (e.g. absolute poverty lines). We show that these indices are affected both by changes in the proportion of poor people and in the share of income held by them. Thus this form of RIC index highlights that pro-poor distributional change can manifest in two different, but related, ways.

A key feature of all these measures is their intuitive interpretation. They reflect changes in the relative situation of the poor vis-a-vis the non-poor: positive values denote a pro-poor development, while negative values reflect anti-poor scenarios.

Finally, our proposals provide a method to acknowledge and quantify potential externalities in the assessment of welfare across people. In our framework, the income growth rate at one particular percentile is not only intrinsically important, but also affects the relative shares of groups defined with different criteria, and therefore perceptions of RIC. The chosen criterion for group partition does affect how the income growth experience of any given percentile contributes to the evaluation of RIC; yet, as we show above, our RIC curves are helpful in pinpointing the circumstances under which the experience of pro-poor (or not) RIC is robust to the varying partitioning criteria.

Acknowledgments We would like to thank Jacques Silber and the anonymous referees for helpful comments and suggestions. Marek Kośny would like to acknowledge the financial support of Polish National Science Centre (Grant No. 3804/B/H03/2011/40). Any remaining errors are our own.

Open Access This article is distributed under the terms of the Creative Commons Attribution 4.0 International License (http://creativecommons.org/licenses/by/4.0/), which permits unrestricted use, distribution, and reproduction in any medium, provided you give appropriate credit to the original 
author(s) and the source, provide a link to the Creative Commons license, and indicate if changes were made.

\section{References}

Acemoglu, D., \& Robinson, J. (2009). Economic origins of dictatorship and democracy. Cambridge: Cambridge University Press.

Ali, I., \& Son, H. (2007). Measuring inclusive growth. Asian Development Review, 24, 11-31.

Atkinson, A. (1970). On the measurement of inequality. Journal of Economic Theory, 2(3), 244-263.

Deutsch, J., \& Silber, J. (2011). On various ways of measuring pro-poor growth. Economics: The OpenAccess Open-Assessment E-Journal, 5, 2011-2013. doi:10.5018/economics-ejournal.ja.2011-13,10. 10.2011.

Dollar, D., \& Kraay, A. (2002). Growth is good for the poor. Journal of Economic Growth, 7, 195-225.

Duclos, J.-Y. (2009). What is "Pro-Poor"? Social Choice and Welfare, 32, 37-58.

Esteban, J., \& Ray, D. (2011). Linking conflict to inequality and polarization. American Economic Review, 101, 1345-1374.

Ferreira, F. (2012). Distributions in motion: Economic growth, inequality, and poverty dynamics, chapter 13. In P. N. Jefferson (Ed.), The oxford handbook of the economics of poverty. Oxford: Oxford University Press.

Foster, J., \& Szekely, M. (2008). Is economic growth good for the poor? tracking low incomes using general means. International Economic Review, 49(4), 1143-1172.

Foster, J., Greer, J., \& Thorbecke, E. (1984). A class of decomposable poverty measures. Econometrica, $52,761-767$.

Grosse, M., Harttgen, K., \& Klasen, S. (2008). Measuring Pro-poor growth in non-income dimensions. World Development, 36(6), 1021-1047.

Kakwani, N., \& Pernia, E. (2000). What is pro-poor growth? Asian Development Review, 18(1), 1-22.

Kakwani, N., \& Son, H. (2008). Poverty equivalent growth rate. Review of Income and Wealth, 54, $643-655$.

Kraay, A. (2006). When is growth pro-poor? Evidence from a panel of countries. Journal of Development Economics, 80(1), 198-227.

Layard, R. (2011). Happiness: Lessons from a new science (2nd ed.). London: Penguin.

Layard, R., Mayraz, G., \& Nickell, S. (2010). Does relative income matter? Are the critics right? chapter 6. In E. Diener, D. Kahneman, \& J. Lelliwell (Eds.), International differences in well-being. Oxford: Oxford University Press.

LIS Web Tabulator (2013). http://www.lisdatacenter.org, Luxembourg, LIS.

Ravallion, M. (2004). Pro-poor growth: a primer. World Bank Policy Research Working Paper, 3242.

Ravallion, M., \& Chen, S. (2003). Measuring pro-poor growth. Economics Letters, 78, 93-99.

Slesnick, D. (1998). Empirical approaches to the measurement of welfare. Journal of Economic Literature, 36, 2108-2165.

Son, H., \& Kakwani, N. (2008). Global estimates of pro-poor growth. World Development, 36(6), 1048-1066.

Temkin, L. (1993). Inequality. Oxford: Oxford University Press.

World Economic Outlook Database (2013). http://www.imf.org/external/ns/cs.aspx?id=28, Washington D.C., International Monetary Fund (14.01.2014).

Zenga, M. (2007). Inequality curve and inequality index based on the ratios between lower and upper arithmetic means. Statistica \& Applicazioni, 1, 3-27. 